\title{
Abstracts of Theses Approved for the MSc and PhD Degrees at the Faculty of Medicine, Health Sciences Centre, Kuwait University, Kuwait
}

\section{MSc Degree}

\author{
1 \\ Immunological Analysis of Dental Pulp Inflammation \\ Mohamed ElSalhy \\ Department of Microbiology, Faculty of Medicine, \\ Kuwait University, Kuwait
}

Assessing the degree of inflammation in the dental pulp poses a diagnostic dilemma as it influences the decision between conservative versus invasive dental treatment. However, there are no objective, quantitative and clinically practical methods for evaluating pulpal inflammation. Cytokines have been suggested to be markers of pulpal inflammation, but estimation of cytokine levels has been possible only after extraction of inflamed teeth. We set out to develop a method to measure cytokines from the dental pulp prior to decision making.

One hundred and eight dental pulp blood samples were obtained with cotton pellets from pulp sites exposed on pulpectomy. Twenty-five samples were from normal teeth, 40 from asymptomatic pulps with caries exposure and 43 from symptomatic pulps clinically diagnosed as irreversible pulpitis. The levels of the inflammatory cytokines IL-2, IL-6, IL-8, TNF- $\alpha$ and IFN- $\gamma$ and the anti-inflammatory cytokine IL-10 were quantified using highsensitivity ELISA. Levels of cytokines and ratios of inflammatory cytokines to IL-10 were compared using Kruskal-Wallis and Mann-Whitney tests.

Significantly higher levels of IL-6, IL-8, IL-10, TNF- $\alpha$ and IFN- $\gamma$ were detected in caries-exposed and irreversible pulpitis as compared to normal teeth. IL-2 levels were higher in caries-exposed as compared to normal teeth. IL-2 and IL-10 levels were higher in caries-exposed pulps as compared to irreversible pulpitis, while IL- 8 was higher in irreversible pulpitis as compared to caries-exposed teeth. Most interestingly, IL-6/IL-10 and IL-8/IL10 ratios were significantly higher in irreversible pulpitis compared to both caries-exposed and normal teeth.
IL-8 levels and the IL-8/IL-10 ratio promise to be good indicators of irreversible pulpitis. An important and potentially very useful outcome of this study is the demonstration that cytokine estimation in pulpal blood may help in the diagnosis of pulpal inflammation.

Supported by a Fellowship from the College of Graduate Studies.

Raj Raghupathy (Supervisor)

Kefah Barrieshi (Co-Supervisor)

2

Detection and Genotyping of Camel Rotaviruses in Kuwait

Laila Zaar Al Mutairi

Department of Microbiology, Faculty of Medicine,

Kuwait University, Kuwait

Rotaviruses are the major cause of acute viral gastroenteritis in neonates and young children worldwide, and in the young animals of a large variety of animal species. Animal rotaviruses have a huge economic and zoonotic importance, as they are a potential reservoir for genetic exchange with human rotaviruses. Camels are economically important animals in the desert regions of North Africa and the Middle East, including Kuwait. Although human and bovine rotaviruses have been incriminated as causes of diarrhea in children and newborn calves in Kuwait, no data are available on the role of rotavirus in camel calves diarrhea. The objective of this study was to detect and characterize rotavirus in camels in Kuwait. Between 2008 and 2010, 408 fecal samples were collected from camels and tested by ELISA kits in 5 geographic areas; only 1 sample was found to be positive for rotavirus. However, 308 serum samples taken from camels revealed that 177 (57\%) samples contained rotavirus antibodies. Serotesting of different age groups of camels for rotavirus-specific antibodies revealed that positive samples were increased after 1 year of age and by 2 years of age, all camels had rotavirus antibodies. Using VP6-

\section{KARGER}

Fax +41 613061234

E-Mail karger@karger.ch

www.karger.com (c) 2012 S. Karger AG, Basel

1011-7571/12/0216-0591\$38.00/0

Accessible online at:

www.karger.com/mpp 
specific RT-PCR, 8 rotavirus strains from 109 randomly selected fecal samples were found in 4 of 5 geographic areas. One of them, $21 \mathrm{~s} / 10$, was genotyped as G10P[15] and this strain is closely related to lamb rotaviruses. This G10 camel rotavirus strain is believed to be the third isolate from camels, and it is the first camel rotavirus with G10P[15] characteristics observed to date. Further studies are needed to collect more detailed and extensive data for the presence of this unique rotavirus genotype and its possible interspecies transmission.

Supported by a fellowship from the College of Graduate Studies and Kuwait University Research Administration grant No. YM 16/08.

Gyorgy Szucs (Supervisor)

Wassim Chehadeh (Co-Supervisor) type I IFNs, whereas very low levels of these cytokines were detected following challenging with CBV2. The clinical CBV1 isolate was a more effective activator of TLR7-induced cytokine production than the laboratory CBV1 strain. In comparison to the enterovirus RNA levels in mock-transfected 293 cells, a reduced virus replication rate was observed in TLR7-transfected 293 cells. A dramatic cytopathic effect was observed in mock- and TLR7transfected 293 cells infected with CBV4, E7, E9 or CBV1 isolate. This cytopathic effect was not associated with high viral load, but with high levels of IL-6, IL- 8 and TNF- $\alpha$. The results suggest a clear segregation of enteroviruses on the basis of their cytokine induction ability, and an enhancement of the enterovirus-induced cytopathic effect by the proinflammatory cytokines.

Supported by a fellowship from the College of Graduate Studies and Kuwait University Research Administration grant No. YM 11/09.

Wassim Chehadeh (Supervisor)

\section{3 \\ Activation of TLR7-Dependent Signaling Pathway in Response to Enterovirus Infection}

Marwa I.A. Alkhabbaz

Department of Microbiology, Faculty of Medicine, Kuwait University, Kuwait

Enteroviruses are single-stranded RNA viruses consisting of more than 60 distinct serotypes associated with many serious diseases. The exact mechanism by which an enterovirus is able to evade innate immunity and cause tissue damage still remains unknown. Toll-like receptors act as the first line of defense of the innate immune system against microbial pathogens. In particular, TLR7 recognizes single-stranded RNA within endosomes, and mediates the activation of type I interferon genes and the development of inflammatory cytokine response. The presence of specific enterovirus strains that can strongly activate or inhibit TLR7-dependent signaling pathway was not yet investigated. Hence, the main aim of the study was to explore the antiviral activity and the pattern of cytokine expression in human embryonic kidney cells (293 cells) expressing TLR7 protein following challenging with enteroviruses. The TLR7 open reading frame insert in the $\mathrm{pENTER}{ }^{\mathrm{TM}} 221$ vector containing the full-length coding sequence of human TLR7 was cloned by recombination reaction into the expression pcDNA3.1/nV5-DEST ${ }^{\text {тм }}$ vector. The 293 cells were transfected with the expression vector, and the expression of TLR7 in 293 cells was confirmed by Western blot. TLR7and mock-transfected 293 cells were infected with different types of laboratory enteroviruses (CBV1-5, E7 and E9), and 2 clinical enterovirus isolates (CBV1 and CBV5). The supernatants of cell cultures were then harvested when a cytopathic effect was first observed in either TLR7- or mock-transfected 293 cells, and the levels of cytokines (IL-6, IL-8, TNF- $\alpha$, IFN- $\alpha$ and IFN- $\beta$ ) were measured by ELISA. The enterovirus RNA concentrations were measured in TLR7- and mock-transfected 293 cell cultures by real-time RT-PCR, and normalized against GAPDH RNA concentrations. The levels of cytokines detected in the supernatants of culture of TLR7-transfected 293 cells were higher than those in the supernatants of culture of mock-transfected 293 cells following challenging with different types of enteroviruses. CBV4 was shown to induce the production of high levels of IL- 6 , IL- 8 and

\section{4 \\ Haptoglobin in Relation to Myeloperoxidase and Prothrombotic Factors in Patients with Thromboembolism}

HebaS.H. Asaad

Department of Pathology, Faculty of Medicine, Kuwait University, Kuwait

Haptoglobin is an acute-phase protein that plays a key role in preventing the oxidative damage of tissues. It can bind to free heme-containing ferric iron to inhibit the accumulation of harmful reactive oxygen species. It has been suggested that haptoglobin polymorphisms contribute to the incidence of thromboembolic diseases; however, mechanisms of association are not fully understood. In addition, prothrombotic coagulation factors have a key role in the development of thromboembolic diseases. Many studies have shown that $\mathrm{Hp} 2-2$ gene polymorphism represents a risk factor for thromboembolic diseases. Myeloperoxidase is associated with acute myocardial infarction and may be used to predict mortality. The main hypothesis in this project is that there is a relation between haptoglobin and haptoglobin gene polymorphism, myeloperoxidase and prothrombotic factors in patients with thromboembolic diseases.

Seventy-two patients with cerebrovascular accident, age range 20-60 years, and 75 healthy controls, age range 30-57 years, are included in this study. Basic hematological and biochemical parameters were estimated. Protein $\mathrm{C}$, protein $\mathrm{S}$, antithrombin, and activated protein $\mathrm{C}$ resistance functional assays were performed. Lupus anticoagulant test was also done. Measuring haptoglobin and myeloperoxidase level was done using ELISA. DNA extraction was done in order to do haptoglobin genotyping. Unfortunately, we faced many methodological problems in determining haptoglobin genotypes and, due to limitation of time, the genotyping could not be accomplished. Our study is the first that tried to find a relation between haptoglobin, myeloperoxidase, and prothrombotic factors in patients with arterial thrombosis. Although we did not find a statistically significant difference in haptoglobin and myeloperoxidase levels between cerebrovascular accident pa- 
tients and controls, cerebrovascular accident patients still had a lower haptoglobin concentration and a higher myeloperoxidase concentration compared to controls. A strong positive correlation was found between haptoglobin and myeloperoxidase, which may be related to their storage site in granulocytes. A positive correlation was found between haptoglobin concentrations and patients' weight. Obesity is a low-grade systemic inflammatory condition that could activate production of haptoglobin and other acutephase proteins. A strong association has been found between age and cerebrovascular accident that may be related to age cumulative effects. A positive correlation was found between protein $S$ and haptoglobin level. This could be due to the fact that protein $S$ is also found in endothelial cells from where it might be released due to endothelial cell injury and atherosclerosis. Haptoglobin can be produced when injury occurs in order to bind free iron and protect endothelium from oxidative stress.

Supported by a fellowship from College of Graduate Studies and Kuwait University Research Administration grant No. 22/09.

Olusegun A. Mojiminiyi (Supervisor)

Rajaa Marouf (Co-Supervisor)

\section{5}

\section{Colorectal Carcinoma in Kuwait: Apoptosis and Its Relation to Clinicopathological Characteristics, p53 Expression and Ki-ras Proto-Oncogene Mutations}

\section{Dana A.A. Al-Qallaf}

Department of Pathology, Faculty of Medicine, Kuwait University, Kuwait

Apoptosis is important in the tumorigenesis of many cancers. Thus, it was hypothesized that the reduction of spontaneous apoptosis has a significant association with advanced colorectal cancer, p53 stability, and Ki-ras mutations. To evaluate the hypothesis, 185 available paraffin-preserved colorectal cancer samples were obtained. Apoptotic index (AI) was evaluated using the terminal deoxynucleotidyl transferase dUTP nick end labeling (TUNEL) assay, while p53 protein expression and Ki-ras mutations were determined using a specific monoclonal antibody and dideoxy sequencing or microarray, respectively. Of the 179 samples that were successfully analyzed, $94(74.1 \%)$ showed p53 protein overexpression, 13 (10.2\%) had Ki-ras mutations, and 20 (15.5\%) had both genetic alterations. In 52 patients (29.1\%), neither p53 overexpression nor Ki-ras mutations were detected. Ki-ras mutations, which were predominantly G $>$ A transitions (67.6\%), were found mostly in codon 12 (91.2\%); codon 13 accounted for the remainder (8.8\%). The $\chi^{2}$ test indicated that high AI was significantly associated with early Dukes' stages ( $\mathrm{p}=$ $0.042)$ and well $/$ moderate tumor differentiation $(\mathrm{p}=0.018)$; high AI was not significantly associated with p53 expression ( $\mathrm{p}=$ $0.920)$ or Ki-ras mutational status $(\mathrm{p}=0.976)$. Dukes' stage was the only factor capable of predicting disease-free survival. Tumors with early Dukes' stage showed a reduced risk of recurrence (hazard ratio, $0.289 ; 95 \%$ CI, 0.122-0.684; $\mathrm{p}=0.005$ ). In conclusion, Dukes' stage remains the best criterion for estimating disease-free survival. Nevertheless, AI appears to differentiate be- tween early and late Dukes' stages, a finding that may have significant clinical relevance.

Supported by a fellowship from the College of Graduate Studies and Kuwait University Research Administration grant No. YM 16/09.

Fahd Al-Mulla (Supervisor)

Salem Al-Shammari (Co-Supervisor)

\section{6}

\section{Polysomy of Chromosome 17: Its Clinicopathological Significance in Breast Cancer Female Patients in} Kuwait

Farah M. Anwar Bakhsh

Department of Pathology, Faculty of Medicine, Kuwait University, Kuwait

Amplification of the HER-2/neu gene is one of the established prognostic markers that leads to protein overexpression in 25$30 \%$ of breast cancer cases. A subset of breast carcinoma showed polysomy of chromosome 17 , which is encountered frequently during the assessment of HER-2/neu status. Unlike gene amplification, the role of polysomy 17 is uncertain. The hypothesis of this study is that polysomy 17 , compared to HER-2/neu-positive and HER-2/neu-negative, has a distinct impact on the clinicopathological features of breast cancer and it can be considered as a useful prognostic marker for breast tumor. We also hypothesized that polysomy 17 is an indirect mechanism that contributes to increased numbers of HER-2/neu gene resulting in protein overexpression in the absence of gene amplification. Therefore, 271 archival, formalin-fixed, paraffin-embedded blocks of breast cancerwere collected.Expressions ofER, PR, Ki-67 and overexpression of HER-2/neu were studied using immunohistochemical method. The FISH assay was used to determine HER-2/neu gene amplification. The frequency of polysomy 17 was $13.6 \%$. In relation to the anatomical site, stage, and histopathological type of the tumor, a statistically significant difference was observed between polysomy 17 and HER-2/neu-negative. In addition, the ER status and PR status of polysomy 17 differed from those of HER-2/neu-positive, and these differences were associated with hormone receptor positivity. Patients with polysomy 17 were also found to have the shortest overall survival $(p=0.001)$ and a trend toward shorter disease-free survival. A highly significant association was observed between polysomy 17 and HER-2/neu protein overexpression $(\mathrm{p}=0.000)$ with a score of $2+$ by immunohistochemistry. These results suggest that polysomy 17 can be characterized as having different clinicopathological features from either HER-2/ neu-positive or HER-2/neu-negative tumors. Our results also showed that polysomy 17 has a significant biological influence on HER-2/neu protein overexpression and that it can be considered as a prognostic marker in breast cancer.

Supported by College of Graduate Studies and Kuwait University Research Administration (grant No. YM 17/09).

Dr. Issam Francis (Supervisor)

Dr. Fahd Al-Mulla (Co-Supervisor) 


\section{7}

\section{Plasma Cell-Free DNA as a Biomarker in Sickle Cell Disease Patients}

Rajaa H. Zureik

Department of Pathology, Faculty of Medicine,

Kuwait University, Kuwait

Sickle cell disease (SCD) is a genetic hemoglobin disorder characterized by the formation of sickled red cells, in which blood becomes viscous and poorly circulates throughout the circulatory system leading to cell death, tissue damage, hemolysis and painful vaso-occlusive crisis (VOC). Because of the repeated cell death and tissue damage, SCD patients are expected to have higher levels of cell-free DNA (cf-DNA) in their plasma. This study investigates plasma cf-DNA concentrations in SCD patients and aims at determining its significance as a biomarker in the diagnosis of SCD. One hundred and forty-eight subjects were recruited from two main hospitals in Kuwait: Mubarak Al-Kabeer hospital and Al-Amiri Hospital. Real-time polymerase chain reaction was used to quantitatively measure $\beta$-globin gene in cell-free blood and urine samples taken from patients with SCD presenting with acute painful VOC, and to compare their cf-DNA levels with steady-state SCD patients and from healthy controls. The results of the study revealed that VOC in Kuwaiti SCD patients led to significantly higher levels of plasma cf-DNA, when compared to the levels in steady-state patients $(\mathrm{p}=0.002)$ and normal controls $(\mathrm{p}=0.0001)$, with no significant change in the levels of urine cfDNA $(p=0.245)$. This result was also supported by our finding of a positive correlation between plasma cf-DNA levels in SCD patients, who are in crisis, and several markers such as WBC counts $(p=0.042)$, serum LDH $(p=0.004), D$-dimers $(p=0.05)$ and CRP $(\mathrm{p}=0.0001)$. The principal conclusion was that the measurement of plasma cf-DNA levels, along with other inflammatory, hemolytic and coagulation activation markers (CRP, WBC, LDH, and $\mathrm{D}$-dimer) are useful in predicting and monitoring the progress of VOC events, as well as in monitoring response treatment in SCD patients.

Supported by Kuwait University, Research Administration grant No. YM23/09.

Salah Al-Humood (Supervisor)

Fahd Al-Mulla (Co-Supervisor)

\section{8 \\ Effect of Alkyl Substituents on the Photosensitizing Activity of Zn(II) N-Alkylpyridyl-Porphyrins}

Mounzer Adnan Ghazal

Department of Biochemistry, Faculty of Medicine,

Kuwait University, Kuwait

The success of photodynamic therapy (PDT), as a minimally invasive approach, in treating both neoplastic and nonneoplastic diseases has stimulated the search for new photosensitizer compounds with potential application in PDT. It has previously been shown that $\mathrm{Zn}$ (II) N-methyl-porphyrins (ZnTM-2-PyP) can act as photosensitizers and kill human carcinoma cells and antibiot- ic-resistant bacteria. It is expected that increasing the hydrophobicity of the photosensitizing molecules will facilitate their cellular uptake, and thus increase their efficacy as photosensitizers. To test this idea, N-butyl and N-hexyl analogs have been synthesized. The aim of the present study was to investigate their photosensitizing activity on eukaryotic cells. The ability of $\mathrm{Zn}$ (II) N-alkylpyridylporphyrins to induce photodynamic damage was tested on a human adenocarcinoma cultured cell line, LS174T. Dark and photo-induced toxicity of $\mathrm{Zn}$ (II) N-alkylpyridylporphyrins were demonstrated by trypan blue exclusion and MTT reduction tests. Mechanisms of photo-induced protein damage were investigated using purified proteins and isolated cell membrane preparations.

The study showed that more lipophilic Zn-metalloalkylpyridylporphyrins gained enhanced photo-cytotoxicity without increased dark toxicity towards cultured cells. These agents caused photo-dependent loss of membrane barrier function and were more efficient in photomodification of membrane proteins than the widely studied photosensitizer hematoporphyrin D. Protein cross-linking activity was reduced, but not abolished, by the presence of singlet oxygen scavengers and these metalloalkylpyridylporphyrins showed capacity for photo-dependent cross-linking of membrane proteins under hypoxic conditions.

These novel lipophilic photosensitizers show potential for future application in PDT.

James Craik (Supervisor)

Ludmil Benov (Co-Supervisor)

\section{9 \\ In vivo Effects of an Environmental Toxicant Lead on Expression of Transcription-Related Proteins and Cell Proliferation Markers in the Liver}

Latifah M.M. Mujaibel

Department of Anatomy, Faculty of Medicine, Kuwait University, Kuwait

The ubiquitous heavy metal lead adversely affects several organ systems. The present study investigated the effects of lead acetate on transcription-related proteins and cell proliferation markers, and compared the effects to the structural changes, oxidative stress status and cell death in the liver. Adult male and female Wistar rats $(13-15$ weeks old; $\mathrm{n}=16)$ were treated with 0.5 and $1 \%$ ( $n=8 /$ group/dose/sample time) lead acetate for 3 days (experiment 1) or 14 days (experiment 2) or 35 days (experiment 3) and sacrificed the next day. The livers were collected and processed for light and electron microscopic evaluations. The total antioxidant status, lipid peroxidation and cell death (TUNEL) were quantified in the liver. The expressions of transcription-related proteins - ERK1/2, p38 $\alpha / \beta$ and JNKs (mitogen-activated protein kinases; MAPK), AP-1 proteins (c-Jun and c-Fos), c-Myc, NF- $\kappa \mathrm{B}$, and cell proliferation markers - PCNA and Ki-67 were investigated by Western blotting and immunohistochemistry or immunofluorescence (confocal microscopy). Data were statistically analyzed by one-way ANOVA followed by the least square difference test. A p value $<0.05$ was considered significant. Lead exposure resulted in a gradual time-dependent increase in structural changes, reduction in total antioxidant status (except on 36 
days), and increase in lipid peroxidation and cell death $(\mathrm{p}<0.05)$. Compared to control livers, the cytoplasmic expression of ERK1/2 decreased (but increased in experiments 2 and 3 at a $1 \%$ dose level). Cytoplasmic and nuclear activities of $\mathrm{p} 38 \alpha / \beta$ (in experiments 1 and 3 with dose- and time-dependent responses in the latter) and JNK (except in $0.5 \%$ groups in experiments 2 and 3 ; dose- and time-dependent responses in experiments 2 and 3 ) increased ( $<<$ $0.05)$. Nuclear expression of AP-1 components was as follows: cJun (except in the $1 \%$ group in experiment 3 ) decreased and c-Fos did not change (but the expression increased in the $1 \%$ group in experiment 3; $\mathrm{p}<0.05)$. Nuclear expression of $\mathrm{c}-\mathrm{Myc}$ decreased ( $\mathrm{p}<0.05$; except in the $0.5 \%$ group in experiment 1 and increased in the $1 \%$ group in experiment 3 with time- and dose-dependent responses) and nuclear and cytoplasmic activities of NF- $\mathrm{KB}$ were unchanged but increased in the $1 \%$ group in experiment 3 ( $\mathrm{p}<$ 0.05). Nuclear expression of PCNA increased in all experiments (dose-dependent effects in experiments 2 and 3 and time-dependent effects in the $1 \%$ group; $\mathrm{p}<0.05$ ) and that of Ki-67 increased in experiments 2 and 3, with time-dependent effects in the latter. In conclusion, lead exposure induces structural changes in a timedependent pattern, but induced oxidative stress and cell death are neither dose- nor time-dependent. Induced changes in MAPK, AP-1, c-Myc, and NF- $\mathrm{B}$ are generally positively correlated with structural changes, oxidative stress and cell death and show doseand time-dependent responses, although not in all groups on all sample times. Despite this, hepatocyte proliferation is upregulated indicating that cell death and cell proliferation coexist in the lead-exposed liver. The results of the present study enhanced our understanding of molecular mechanisms underlying lead-induced hepatotoxicity and pave the way for further research on counteracting strategies for lead toxicity.

Supported by College of Graduate Studies and Kuwait University grant No. YS05/09 and GM01/01 and GM01/05.

Narayana Kilarkaje (Supervisor)

\section{PhD Degree}

1

\section{Health-Related Physical Activity Survey of Kuwaiti Adult Population: Experimental Validation and Application of a Recall Instrument}

\author{
Nadia H.J. Garashi
}

Department of Physiology, Faculty of Medicine, Kuwait

University, Kuwait

Introduction: Regular physical activity (PA) is linked to physical and mental health benefits. Low levels of PA are becoming an evolving problem in Kuwait and worldwide. The need for surveillance requires valid, reliable, and preferably comparable tools that can estimate the level of PA in a society. The aim of this study, therefore, was to establish a standard PA surveillance tool in which the International Physical Activity Questionnaire (IPAQ) is incorporated as the PA-measuring tool, in our Kuwait Physical Activity Questionnaire (KPAQ). Methods: In phase 1, testing of the KPAQ was conducted $(\mathrm{n}=145)$ involving translation, backtranslation, cultural adaptation, and testing of the IPAQ. It served to evaluate appropriateness of the KPAQ, logistics of distribution, data entry and analysis. Phase 2 involved test-retest reliability assessment $(\mathrm{n}=71)$, where subjects repeated the KPAQ within 6-8 days after initial administration. Criterion validity $(n=40)$ was also done to compare IPAQ data with data from the Intelligent Device for Energy Expenditure and Activity (IDEEA). In phase 3, a population survey $(n=1957)$ was conducted using this reliable and valid KPAQ in a representative sample of Kuwaitis aged 17-65 years. Results: For reliability, the correlation coefficient (r) for IPAQ items reflecting PA in min/week ranged between 0.63 and 0.74 , and was 0.79 for total PA MET min/week. In criterion validity, MET min/week from IDEEA and IPAQ correlated significantly $\left(r=0.61 ; r^{2}=0.38\right)$. For the survey, categorical IPAQ scores showed that $33.7 \%$ had a high, $36.9 \%$ a moderate, and $29.4 \%$ a low PA level. Males (especially singles and students) and regularly exercising individuals more likely had a high PA level. Females more likely had moderate PA levels than males. $37.1 \%$ performed moderate PA for at least 150 min per week. The percentage of regularly exercising individuals was $47.3 \%$, higher in males than females (58 compared to $39.8 \%$; $p<0.001$ ). The prevalence of selfreported chronic diseases was high, considerably increasing with age. Low PA level and overweight/obesity were highly prevalent even in the youngest age group. Conclusions: The high percentage of Kuwaitis who failed to reach a health-enhancing PA level may be related to the elevated incidence of chronic diseases in Kuwait. The KPAQ appears to be a reliable and valid tool for surveillance of PA.

Jasem Ramadan (Supervisor) 